3 Bo Dong ${ }^{1,11}$, Xiaoqian $\mathrm{Lin}^{2,3,4,5,11}$, Xiaohuan $\mathrm{Jing}^{3,11}$, Tuanyuan $\mathrm{Hu}^{4,11}$, Jianwei 4 Zhou $^{2,6}$, Jianwei Chen ${ }^{2,6,7}$, Liang Xiao ${ }^{2,4,7,8}$, Bo Wang ${ }^{3,9}$, Zhuang Chen ${ }^{1,10}$, Jing $5 \quad \mathrm{Liu}^{1,10}$, Yiyin $\mathrm{Hu}^{3}$, Guilin $\mathrm{Liu}^{6}$, Shanshan $\mathrm{Liu}^{2,6}$, Junnian Liu ${ }^{2,6}$, Wenkang Wei ${ }^{1,10^{*}}$, 6 Yuanqiang $\mathrm{Zou}^{2,4,7,8^{*}}$

$8{ }^{1}$ Guangdong Provincial Key Laboratory for Crop Germplasm Resources Preservation 9 and Utilization, Agro-biological Gene Research Center, Guangdong Academy of

$11{ }^{2}$ Qingdao-Europe Advanced Institute for Life Sciences, BGI-Shenzhen, Qingdao 12 266555, China

$13{ }^{3}$ China National Genebank, BGI-Shenzhen, Shenzhen 518120, China

$14{ }^{4}$ BGI-Shenzhen, Shenzhen 518083, China

$15{ }^{5}$ School of Bioscience and Biotechnology, South China University of Technology, 16 Guangzhou 510006, China

$17{ }^{6}$ BGI-Qingdao, BGI-Shenzhen, Qingdao 266555, China

$18{ }^{7}$ Laboratory of Genomics and Molecular Biomedicine, Department of Biology, 19 University of Copenhagen, Universitetsparken 13, 2100 Copenhagen, Denmark

$20{ }^{8}$ Shenzhen Engineering Laboratory of Detection and Intervention of human intestinal 21 microbiome, BGI-Shenzhen, Shenzhen, China

$22{ }^{9}$ Guangdong Provincial Key Laboratory of Genome Read and Write, BGI-Shenzhen, 23 Shenzhen, 518120, China

$24{ }^{10}$ Guangdong Laboratory for Lingnan Modern Agriculture, Guangzhou, Guangdong 25 510642, China 
$26{ }^{11}$ These authors contributed equally: Bo Dong, Xiaoqian Lin, Xiaohuan Jing, and

27 Tuanyuan $\mathrm{Hu}$.

\section{$29{ }^{*}$ Corresponding authors:}

30 Wenkang Wei.

31 Address: Innovation Building, Agrogene-Centre, GDAAS, Guangzhou 510640,

32 China.

33 E-mail: weiwenkang@gdaas.cn

34 Yuanqiang Zou.

35 Address: BGI-Shenzhen, Beishan Industrial Zone, Shenzhen 518083, China.

36 E-mail: zouyuanqiang@genomics.cn 
ABSTRACT

The microbiota hosted in the pig gastrointestinal tract are important for

51 productivity of livestock. However, the individual species and functional repertoires that make up the pig gut microbiome remain largely undefined. Here we comprehensively investigated the genomes and functions of the piglet gut microbiome using culture-based and metagenomics approaches. A collection included 266 cultured genomes and 482 metagenome-assembled genomes (MAGs) that were clustered to 428 species across 10 phyla was established.

57 Among these clustered species, 333 genomes represent potential new species. Less matches between cultured genomes and MAGs revealed a substantial bias

59 for the acquisition of reference genomes by the two strategies. Glycoside

60 hydrolases was the dominant category of carbohydrate-active enzymes. 445

61 secondary metabolite biosynthetic genes were predicted from 292 genomes with

62 bacteriocin being the most. Pan genome analysis of Limosilactobacillus reuteri

63 uncover the biosynthesis of reuterin was strain-specific and the production was 64 experimentally determined. These genomic resources will enable a 65 comprehensive characterization of the microbiome composition and function of 66 pig gut.

69 Pigs are economically important livestock, widely used as monogastric animal 70 model for enteric microbiological studies, and serve as most consumed meat for 71 human worldwide ${ }^{1,2}$. A large amount of microorganisms harbored in the gut of pig.

72 The research of gut microbiome of pig has increasingly advanced our understanding 73 of the role of the microbiota in feed conversion efficiency, pig health, and 74 production in recent years ${ }^{3,4}$. 
75 Metagenomic studies have uncovered the genes, species, and functional diversity of 76 bacteria in mammalian intestines with the advantage in avoiding the time-consuming 77 of traditional culture-based approach and the inaccuracy of 16S rRNA sequences 78 analysis. A gene catalog consisting of 7.7 million non-redundant genes has been 79 constructed that draw a basic function map of the pig gut microbiota ${ }^{5}$. PIGC, the pig 80 integrated gene catalog, a recent expanded gene catalog comprised 17 million 81 complete genes that provide an expanded resource for pig gut microbiome research ${ }^{6}$.

82 Metagenomic binning is an essential tool for acquiring reference genomes readily, 83 particularly for uncultured microbiota, that has been widely used in study of human 84 gut microbiota, but rarely applied for pigs. However, misassembles and chimeric 85 contigs from MAGs result in substantial biases for the analysis of the gut 86 microbiome $^{7,}{ }^{8}$. These metagenomic studies have greatly increased our 87 understanding of pig gut microbiome $e^{6,9}$. Nevertheless, the lack of bacterial isolates 88 and high-quality genomes limited our comprehensively understanding of the 89 structure and function of the gut microbiota of pig and the development of probiotics 90 for pig farming.

91 The culture-based approach has been well used for establishment of bacterial 92 collection for the human gut microbiota ${ }^{10}$. Over 1,500 species have been 93 successfully isolated from the human gut by using culturomics, introduced by Lagier 94 et al. and 247 new species have been unveiled ${ }^{11}$. Culture-based studies enable the 95 acquisition of both live bacteria and high-quality reference genomes and provide 96 experimental access for function exploration and intervention trials with probiotics.

97 PiBAC, a pig intestinal bacterial collection, was constructed by cultivation of 110 98 species and described 38 novel species from the gut of 19 pigs in Germany, United 99 States, and Canada ${ }^{12}$. This study highlighted the importance and necessity of 100 continuously isolating and characterizing microbial taxa from pig gut.

101 Here we performed a study of bacterial cultivation and genome sequencing along with 102 deep metagenome sequencing of 14 samples, including ileum and colon contents, and 103 feaces, from weanling piglets. A total of 266 isolated bacterial genomes and 482 
104 MAGs were obtained and investigated their functional repertoires. This study

105 provides a comprehensive view of the microbiome composition and the function

106 landscape of the gut of weanling piglets and a valuable bacterial resource for further

107 experimentations.

108

\section{RESULTS}

110 The metagenome sequencing described community composition of microbiota in 111 different gut regions of weanling piglets

112 A total of 42 samples, including 15 ileum contents, 18 colon contents and 9 faeces, 113 were collected from 42 weanling piglets from Guangdong Academy of Agricultural 114 Sciences. Every 3 samples from one line were combined into one sample which 115 ultimately resulted in 14 samples. To investigate the microbiota composition in each 116 sample, we first performed deep metagenomic sequencing for these samples and 117 generated $20 \mathrm{~Gb}$ of data for each sample on average. Notably, over $12 \%$ of reads 118 mapped to bacteria that could not be classified at the species level, which represent 119 novel species in the gut of weanling piglets (Supplementary Fig. 1). 99.8\% of the 120 bacterial reads were assigned into 10 phyla. The phylum Firmicutes dominated the

121 gut microbiota in the ileum, colon, and faeces, accounting for $98.9 \%, 67 \%$, and $76.8 \%$

122 of the bacterial sequences, respectively (Figs. 1a, b). We also discovered

123 Lactobacillus were common present in 3 regions and accounted for the highest

124 proportion in samples from faeces and ileum and some samples from colon

125 (Supplementary Fig. 2a, b). The higher microbial diversity, including the Shannon

126 index, was observed in the colon as compared with the faeces and ileum (Fig. 1c),

127 consistent with previous studies ${ }^{13,14}$. The principal coordinates analysis showed that

128 the microbiota composition of the faeces and colon were similar and were different

129 from that of the ileum (Fig. 1d). These results indicated the regional similarities and

130 differences in microbiota composition and the beneficial bacteria, like Lactobacillus

131 dominated the gut microbiota of weanling piglets, were valuable resource for the 
132 furtherly cultured-based study.

\section{Bacteria culturing and genome sequencing of gut of weanling piglets}

134 To advance our understanding of the diversity of gut microbiota and acquire bacterial

135 isolates from the weanling pig gut, we used a variety of culture methods, including 136 nutrient-rich medium (MPYG, with or without sheep blood), oligotrophic medium 137 (R2A), Columbia Agar, spore medium, etc. In total, 25 culture conditions were used 138 (Supplementary method). Most of the bacteria were cultured in sheep blood-MPYG, 139 MPYG, and sheep blood-spore medium. A total of 1,476 strains were obtained and 140 identified using 16S rRNA gene sequence, the detail information is provided in 141 Supplementary Table 2. These isolates belonged to 5 different phyla, including 142 Firmicutes, Bacteroidetes, Actinobacteria, Proteobacteria, and Fusobacteria 143 (Supplementary Fig. 3). Limosilactobacillus reuteri was the most abundant, which 144 represented $18 \%$ of the taxa isolated and half of these 1,476 bacteria were 145 Lactobacillus (recently divided as Lactobacillus, Ligilactobacillus, and 146 Limosilactobacillus), representing the beneficial bacteria in gut of piglets 147 (Supplementary Fig. 4). Most of these bacteria were the first time isolated from the 148 pig gut microbiota, which expanded the bacteria diversity of the gut microbiota of pig.

149 Notably, the isolated bacteria from the ileum, colon, and faeces were much different 150 with highest species diversity of bacteria from faeces sample, that consistent with the 151 analysis of metagenome (Supplementary Fig. 4). All bacteria have been deposited in 152 the China National Genebank (CNGB) Shenzhen for public accessibility.

\section{Comparison of cultured genomes and MAGs}

154 We selected 266 representative strains that covered the bacterial diversity of the 1,476 155 isolates for genome sequencing. After assembly, 266 high-quality genomes with 156 completeness more than $90 \%$ and contamination less than $5 \%$ were obtained 157 (Supplementary Table 3a), of which 195 genomes (73.31\%) were more than 99\% 158 completeness (Supplementary Fig. 5a), indicating that the great majority of the 159 genomes were relatively complete. Based on the $95 \%$ average nucleotide identity 
160 (ANI), we clustered the 266 genomes into 59 species-level clusters. The vast majority

161 of clusters were Firmicutes (242 genomes, 52 clusters), followed by Proteobacteria

162 (18 genomes, 3 clusters), Actinobacteriota (4 genomes, 2 clusters), Bacteroidota (1

163 genome, 1 cluster) and Fusobacteriota (1 genome, 1 cluster) according to the

164 taxonomic annotation of these genomes (Fig. 2a). Notably, 10 out of 59 clusters (23

165 genomes) lacked a species-level match with the GTDB-Tk database (Fig. 2a and

166 Supplementary Table 3a).

167 By Assembling and binning metagenomic sequence data from 14 samples, we 168 reconstructed 482 non-redundant MAGs with completeness more than $70 \%$ and 169 contamination less than 5\% (Supplementary Table 3b). Only 223 (46.27\%) MAGs

170 were high-quality genomes (completeness more than 90\%), 11 of which with 171 completeness more than 99\% (Supplementary Fig. 5b), which indicated that the 172 quality of the genomes generated by culture-based methods was generally higher than 173 metagenomic assembly. To evaluate the species bias caused by the two methods, we 174 clustered 489 high-quality genomes. The results showed that MAGs and isolated 175 genomes had $74.63 \%$ and $25.42 \%$ of unique species-clusters, respectively 176 (Supplementary Fig. 6a, b), illustrating that the combination of two methods is 177 needed to more comprehensively represent the species of the pig gut microbiota. We 178 thus clustered the 748 genomes into 428 species-clusters to integrate the reference 179 genomes. According to the GTDB classification annotations, 5 clusters (5 MAGs) 180 were Archaea, and the remaining clusters were bacteria from 10 phyla (Fig. 2a and 181 Supplementary Table 4). It is worth noting that $77.80 \%$ of clusters (333 clusters) 182 could not be matched with any existing species, which represent novel species, and $18396.70 \%$ (322 clusters) of the novel species without isolate genome representative in 184 this study (Fig. 2a).

185 Previous studies have used metagenomics or culture-based method to study the 186 intestinal microbes of pigs. Wang et al. ${ }^{9}$ generated 360 high-quality assembled 187 genomes for pig fecal microbiome. And Wylensek et al. ${ }^{12}$ established the pig 
188 intestinal bacterial collection (PiBAC), a resource of cultured bacteria from the pig

189 intestine. We evaluated the novelty of our genomes by mapping 748 genomes

190 against these two reference datasets. The result showed that our MAGs and isolated 191 genomes had $65.89 \%$ and $50.85 \%$ unique clusters (novel species), respectively (Fig.

192 2b, c and Supplementary Fig. 7a, b), which contribute new resources for the research 193 of pig gut microbiota. In addition, most of the clusters were only detected in the

194 MAG dataset, but not in any culture studies, reflecting the lack of culture-based 195 studies of pig intestines.

\section{The assembled genes contributed to the present gene catalog}

197 We next predicted genes from the 14 metagenomic samples and generated a 198 non-redundant gene catalog with a number of $5,283,405$, which covered $22.01 \%$ of 199 the reference gene catalog established by Xiao et al. ${ }^{5}$ (Fig. 3a, b). The samples from 200 faeces contained the most genes, followed by the colon and ileum. Each sample 201 contributed more than 50\% of novel genes for the gene catalog. Among them, 202 samples from ileum have the largest proportion of novel genes, followed by colon 203 and faeces (Fig. 3a). Considering the samples of the reference gene catalog by Xiao 204 et al. were derived from pig faeces, we thought that construct a more complete gene 205 catalog of pig intestinal needs to include samples from multiple parts. Altogether, we 206 expanded the present gene catalog to $10.91 \mathrm{Mb}$ (Fig. 3b). In addition, the coverage 207 of genes obtained by MAGs and isolated genomes were $12.97 \%$ and $1.29 \%$, 208 respectively, whereas $62.97 \%$ of the genes in the isolated genomes could not be 209 detected by metagenomics (Fig. 3c). This indicates that the missed genes from 210 metagenomic analyses can be detected by culture-dependent methods.

\section{Functional insight of gut microbial in weanling piglets}

212 The comprehensive gene catalog of pig intestines enables a higher-resolution

213 functional analysis to better understand the interaction between gut microbes and

214 pigs. We subsequently performed a KEGG pathway annotation of total protein 215 sequences and found that 213 pathways were annotated in at least one genome. The 
216 most general pathway was Metabolic pathways, followed by Biosynthesis of

217 secondary metabolites, Biosynthesis of antibiotics, Microbial metabolism in diverse

218 environments, and Biosynthesis of amino acids, which were extensively annotated in

219 all genomes (Supplementary Fig. 8 and Supplementary Table 5). In addition, we

220 predicted 229 carbohydrate-active enzymes (CAZymes) in all genomes to explore

221 the ability to metabolize carbohydrates (Fig. 4a and Supplementary Table 5). The

222 result showed that the CAZymes of bacteria are mainly glycoside hydrolases (GHs),

223 whereas archaea are glycosyl transferases (GTs) (Fig. 4a). This indicates that

224 bacteria obtained energy for growth by metabolizing carbohydrates, while archaea

225 obtain energy through other pathways and synthesize sugars substance.

226 Antibiotics are widely used for the treatment and prevention of infection during the 227 suckling period. Microbes may accumulate antibiotic resistance genes (ARGs) under 228 the exposure of antibiotics, and can eventually evolve into drug resistance. We have 229 predicted a total of 2,113 ARGs that can be classified into 38 drug classes from 230 metagenomics, isolate genomes and MAGs (Fig. 4b). Comparison of the ARGs 231 predicted by the three approaches showed that metagenomics can detect the largest 232 number and types of ARGs, followed by culture-dependent, while a large number of 233 ARGs will be lost in the process of reconstructed genomes from metagenomic bins 234 (Fig. 4b). For analysis of virulence factors (VFs), we used the predicted genes for 235 blast to $\mathrm{VFDB}^{45}$. A total of $1,866 \mathrm{VFs}$ were predicted in 748 genomes 236 (Supplementary Fig. 9). Similarly, a large amount of VFs cannot be detected in MAG. 237 We note that Escherichia flexneri not only had most various ARGs, but also 238 contained a large number of VFs.

\section{Discovery of novel SMBGs in the gut microbiome of weanling piglets}

240 Microbes produce a series of secondary metabolites that are not necessary for life 241 activities but have biological activity, which usually mediate important interactions 242 between microbe and microbe-hosts. We explored secondary metabolite biosynthetic 243 gene clusters (SMBGs) in the 748 genomes, and identified 445 SMBGs that could 244 be classified into 19 types from 292 genomes (Supplementary Table 6). Most of 245 these SMBGs are responsible for the synthesis of bacteriocin, followed by 246 sactipetide, arylpolyene, and lantipeptide (Fig. 4c). These SMBGs are predicted 
247 from the genomes from 6 phyla, among which Firmicutes contained the largest

248 number and widest variety of SMBGs (Fig. 4c).

249 For clustering the 445 SMBGs, we generated a total of 231 families, which were

250 distributed in 5 classes of ribosomally synthesized and post-translationally modified

251 peptides (RiPPs), terpene, non-ribosomal peptide synthetases (NRPs), polyketide

252 synthases (PKS) and Others. RiPPs make up the largest class, but only 4 families of

253 RiPPs that are included the reference with known functions in the MIBiG database.

254 Salivaricin CRL1328 is a bacteriocin produced by Ligilactobacillus salivarius ${ }^{15,16}$,

255 which has activity against pathogenic bacteria such as Enterococcus faecalis and

256 Enterococcus faecium. We have predicted SMBGs related to the biosynthesis of

257 Salivicin CRL1328 in 22 isolated L. salivarius genomes (Fig. 4d). We speculating

258 that these strains have the potential to inhibit infections. Gassericin, derived from

259 Lactobacillus gasseri, is another important bacteriocin. Studies have shown that

260 Gassericin A can confer diarrhea resistance in pigs ${ }^{17}$. We discovered SMBGs that

261 synthesize Gassericin E and Gassericin T in isolated genomes of L. johnsonii (Fig. $2624 d)$.

\section{The pangenome analysis of representative species}

264 To extend the phylogenetic analysis of representative species in the gut of weanling 265 piglets at a genome-wide level, we conducted a pangenome analysis of 8 species 266 with the genome number more than 10, including Escherichia flexneri $(\mathrm{n}=16)$, 267 Enterococcus faecalis $(\mathrm{n}=22)$, Enterococcus faecium $(\mathrm{n}=21)$, which represent 268 opportunistic pathogens, and Lactobacillus amylovorus ( $\mathrm{n}=16)$, Ligilactobacillus 269 salivarius $(\mathrm{n}=43)$, Limosilactobacillus mucosae $(\mathrm{n}=13)$, Limosilactobacillus reuteri 270 ( $\mathrm{n}=21)$, Lactobacillus johnsonii $(\mathrm{n}=17)$, which represent probiotics. As expected, the 271 resulting accumulation curves showed that the gene repertoires of pan-genome of all 272 the representatives were increased on addition of a new genome and the core 273 genome decreased in contrast (Fig 5a and Supplementary Fig. 10). However, the 274 number of gene families did not show a rapid increase in the pan genome. E. flexneri 
275 contained the largest pan genome size of 8,143 genes, 3,111 of which formed the 276 core genome (Supplementary Fig. 11 and Supplementary Table 7). Over half of core 277 genes present in the genomes of E. faecalis and E. faecium, indicating that gene loss 278 and acquisition happened less frequently in these two species. The size of the 279 species-specific pan genomes of the 5 lactobacilli varied from 3,194 to 4,187 genes, 280 respectively (Supplementary Fig. 11 and Supplementary Table 7).

281 We next analyzed the function details of the core genomes and pan genomes, 282 including the Clusters of Orthologous Groups (COGs) and KEGG classification, 283 biosynthesis of bacteriocin, and ARGs. It is obvious that different COG functional 284 classes were enriched in both the core and pan genomes between the opportunistic 285 pathogens and probiotics. COG0438 (Glycosyltransferase involved in cell wall 286 biosynthesis), COG4690 (Dipeptidase), and COG1307 (Fatty acid-binding protein $287 \mathrm{DegV}$ ) were enriched in the core genomes compared to their respective accessory 288 and unique genomes of the 5 lactobacilli (Supplementary Table 8), indicating that 289 these COG categorizations represented housekeeping functions involved in 290 implementing the basic growth and metabolism of these probiotics. However, in 291 contrast to lactobacilli, E. flexneri, E. faecalis, and E. faecium possessed more 292 abundant COG1609 (DNA-binding transcriptional regulator, LacI/PurR family) and 293 COG1132 (ABC-type multidrug transport system, ATPase and permease component) 294 in their core genomes (Supplementary Table 8).

295 For determining the ARGs distribution in the core and pan genome of the 8 species, 296 we discovered tetracycline and macrolide were distributed in the core genome of $E$. 297 flexneri, E. faecalis, and E. faecium, respectively (Fig. 5b), indicating a wide 298 prevalence of tetracycline and macrolide resistance amoung these species. Their 299 presence may have resulted from the overuse of antibiotics in farmed pigs for 300 disease prevention in past years. Nevertheless, rare ARGs present both in the core 301 and pan genome of other lactobacilli (Fig. 5b and Supplementary Table 9), suggests 302 the safety of these species for using as probiotics in feeding of weanling piglet. 
303 Reuterin, produced by some strains of L. reuteri, has antimicrobial properties ${ }^{18}$. We

304 investigated the prevalence of biosynthesis genes (pdu-cbi-cob-hem) related to

305 reuterin in the core and dispensable genomes and detected the production of reuterin

306 experimentally. In total 41 genes related to the synthesis of reuterin were predicted

307 in the pan genome of L. reuteri, including cbiA cbiT (15 genes), cobB cobU (10

308 genes), hemA hemL (7 genes), and $p d u A \sim p d u V$ (9 genes) (Fig. 5c and

309 Supplementary Table 10). Notably, only cobB, cobC_1, and $c o b C \_2$ were found in

310 the core genomes for all the 21 genomes. The rest were present in the accessory or

311 unique genomes. 9 of 21 genomes encoded completed biosynthesis gene clusters

312 ( $p d u$-cbi-cob-hem), indicating these strains were most likely to be reuterin producers.

313 We next detected the production of Reuterin in the fermentation supernatant of the

314 culture of 19 isolated L. reuteri. As expected, 4 strains with completed biosynthesis

315 gene clusters (pdu-cbi-cob-hem) yield reuterin ranged from 0.6 to $2.54 \mathrm{~nm} / \mathrm{L}$

316 (Supplementary Table 10).

\section{DISCUSSION}

319 Previous culture-independent methods have uncovered that the gut microbiota is

320 associated with the survival rate of piglet after weaning ${ }^{19}$. In this study, we

321 investigated the gut microbiota of piglets combining with culture-based and 322 metagenomic strategies. A collection including 1,476 cultured bacteria, 748 323 reference genomes catalog, comprising 266 cultured genomes and 482 MAGs, 324 which represent the gut microbiome of piglet was constructed. $70.80 \%$ of these were 325 represent novel species according to the present database. We also found 5 MAGs 326 were archaea. We contributed 3,630,043 novel genes to the present pig gene catalog 327 and expanded to $10.91 \mathrm{Mb}$. In our collection, over half of the taxa were lactobacilli, 328 that will be a valuable resource for probiotics development and diversity study for 329 different niche of these species.

330 We discovered $84.20 \%$ of the clusters from MAGs could not be assigned to any 
331 known species, only 3 clusters matched 11 cultured genomes with novelty.

332 Prevotella, a dominant genus in the gut of piglet, can be detected using 333 metagenomic analysis and a total of 26 genomes from the metagenome bins were 334 annotated as Prevotella, but none were included in our culture collection constructed 335 in this study. This emphasizes the necessity of continuing effort for developing more 336 culture methods that are appropriate for more bacteria.

337 Our genome-based functional analysis provides a better understanding of the gut 338 microbiota of weanling piglets. Genes related to the metabolic pathways, 339 biosynthesis of secondary metabolites, and Biosynthesis of antibiotics were highly 340 enriched in all genomes. We found that the gut microbiota of weaning piglets 341 contained a large number of glycoside hydrolase enzymes, which can be used to 342 metabolize major dietary carbohydrates such as corn, soybean meal, wheat, and rice 343 bran to provide most of the energy and nutrients needed for daily life ${ }^{20}$. The 344 excavation of SMBGs found antibiotics that inhibit pathogenic bacteria such as $E$. 345 faecalis and E. faecium, emphasized the effect of gut microbiomes in suppressing 346 infections in piglets. Finally, we annotated the ARGs and VFs from metagenomes 347 and genomes. It is worth noting that the metagenomes contained abundant ARGs 348 and VFs, which were distributed in specific isolated genomes, whereas the MAGs 349 had a very small number of annotated ARGs and VFs. We highlighted that both 350 techniques are complement for the detection of harmful genes.

351 Pan genome analyses provide comprehensive genetic landscapes of the 352 representative species and identify the characteristics of core and strain-specific 353 genes. Lactobacillus, which represent the probiotics in the pig gut, implement 354 functions with COG0438 (Glycosyltransferase involved in cell wall biosynthesis), 355 COG4690 (Dipeptidase), and COG1307 (Fatty acid-binding protein DegV). 356 Bacteriocin, like reuterin, can be used as a broad-spectrum antimicrobial agent to 357 prevent piglet diarrhea ${ }^{17}$. We discovered the completeness of biosynthesis pathway 358 for reuterin is strain-specific, the reuterin-related genes were present in the 359 dispensable genome, indicating when exploitation and application of bacteriocin, the 
360 strain level diversity should be considered. In this collection, we identified 4/21 L.

361 reuteri stains that had the capacity to produce reuterin, indicating those strains have

362 potential in prevention of pathogen infections and colonization of the piglet 363 gastrointestinal tract.

364 Metagenomic binning has been widely used to recover genomes from the fecal 365 samples. But the cultured bacteria are of great importance for the experimental 366 validation of their functions, especially for the development of probiotics. As a 367 result of this study, we have provided both the cultured bacteria and reference 368 genome data, that are publicly available at CNGB. It will be a useful resource for the 369 future studies of microbiota-host interactions and the development as probiotics for 370 disease prevention of weanling piglets.

\section{REFERENCES}

372 1. Maes DGD, Dewulf J, Pineiro C, Edwards S, Kyriazakis I. A critical 373 reflection on intensive pork production with an emphasis on animal health 374 and welfare. J Anim Sci 98, S15-S26 (2020).

375 2. Chen M, Hu E, Kuen LL, Wu L. Study on Consumer Preference for 376 Traceable Pork With Animal Welfare Attribute. Front Psychol 12, 675554 377 (2021).

378 3. McCormack UM, et al. Porcine Feed Efficiency-Associated Intestinal 379 Microbiota and Physiological Traits: Finding Consistent Cross-Locational 380 Biomarkers for Residual Feed Intake. mSystems 4, e00324-18 (2019).

381 4. Torres-Pitarch A, et al. Effect of cereal fermentation and carbohydrase 382 supplementation on growth, nutrient digestibility and intestinal microbiota in 383 liquid-fed grow-finishing pigs. Sci Rep 10, 13716 (2020).

384 5. Xiao L, et al. A reference gene catalogue of the pig gut microbiome. Nat $385 \quad$ Microbiol 1, 16161 (2016).

386 6. Chen C, et al. Expanded catalog of microbial genes and 387 metagenome-assembled genomes from the pig gut microbiome. Nat $388 \quad$ Commun 12, 1106 (2021).

389 7. Teeling H, Glockner FO. Current opportunities and challenges in microbial 
metagenome analysis--a bioinformatic perspective. Brief Bioinform 13, 728-742 (2012).

8. Howe A, Chain PS. Challenges and opportunities in understanding microbial communities with metagenome assembly (accompanied by IPython Notebook tutorial). Front Microbiol 6, 678 (2015).

9. Wang W, Hu H, Zijlstra RT, Zheng J, Ganzle MG. Metagenomic reconstructions of gut microbial metabolism in weanling pigs. Microbiome $\mathbf{7}$, 48 (2019).

10. Lagier JC, et al. Culturing the human microbiota and culturomics. Nat Rev Microbiol 16, 540-550 (2018).

11. Lagier JC, et al. Culture of previously uncultured members of the human gut microbiota by culturomics. Nat Microbiol 1, 16203 (2016).

12. Wylensek D, et al. A collection of bacterial isolates from the pig intestine reveals functional and taxonomic diversity. Nat Commun 11, 6389 (2020).

13. Kim HB, Isaacson RE. The pig gut microbial diversity: Understanding the pig gut microbial ecology through the next generation high throughput sequencing. Vet Microbiol 177, 242-251 (2015).

14. Li K, Xiao Y, Chen J, Chen J, He X, Yang H. Microbial composition in different gut locations of weaning piglets receiving antibiotics. Asian-Australas J Anim Sci 30, 78-84 (2017).

15. Ocana VS, Pesce De Ruiz Holgado AA, Nader-Macias ME. Characterization of a bacteriocin-like substance produced by a vaginal Lactobacillus salivarius strain. Appl Environ Microbiol 65, 5631-5635 (1999).

16. Vera Pingitore E, Hebert EM, Nader-Macias ME, Sesma F. Characterization of salivaricin CRL 1328, a two-peptide bacteriocin produced by Lactobacillus salivarius CRL 1328 isolated from the human vagina. Res Microbiol 160, 401-408 (2009).

17. $\mathrm{Hu} \mathrm{J}$, et al. A Microbiota-Derived Bacteriocin Targets the Host to Confer Diarrhea Resistance in Early-Weaned Piglets. Cell Host Microbe 24, 817-832 e818 (2018).

18. Zhang Z, et al. A Phylogenetic View on the Role of Glycerol for Growth Enhancement and Reuterin Formation in Limosilactobacillus reuteri. Front 
Microbiol 11, 601422 (2020).

423

424

425

426

428

430

432

439

440

442

444

445

447

450

451

452

19. Gresse R, Chaucheyras-Durand F, Fleury MA, Van de Wiele T, Forano E, Blanquet-Diot S. Gut Microbiota Dysbiosis in Postweaning Piglets: Understanding the Keys to Health. Trends Microbiol 25, 851-873 (2017).

20. Lindberg JE. Fiber effects in nutrition and gut health in pigs. J Anim Sci Biotechnol 5, 15 (2014).

21. Guo X, et al. CNSA: a data repository for archiving omics data. Database (Oxford) 2020, (2020).

22. Chen FZ, et al. CNGBdb: China National GeneBank DataBase. Yi Chuan 42, 799-809 (2020).

23. Zou Y, et al. 1,520 reference genomes from cultivated human gut bacteria enable functional microbiome analyses. Nat Biotechnol 37, 179-185 (2019).

24. Zou Y, et al. Lactobacillus shenzhenensis sp. nov., isolated from a fermented dairy beverage. Int J Syst Evol Microbiol 63, 1817-1823 (2013).

25. Yarza P, et al. Uniting the classification of cultured and uncultured bacteria and archaea using 16S rRNA gene sequences. Nat Rev Microbiol 12, 635-645 (2014).

26. Katoh K, Standley DM. MAFFT multiple sequence alignment software version 7: improvements in performance and usability. Mol Biol Evol 30, 772-780 (2013).

27. Capella-Gutierrez S, Silla-Martinez JM, Gabaldon T. trimAl: a tool for automated alignment trimming in large-scale phylogenetic analyses. Bioinformatics 25, 1972-1973 (2009).

28. Price MN, Dehal PS, Arkin AP. FastTree 2--approximately maximum-likelihood trees for large alignments. PLoS One 5, e9490 (2010).

29. Chen Y, et al. SOAPnuke: a MapReduce acceleration-supported software for integrated quality control and preprocessing of high-throughput sequencing data. Gigascience 7, 1-6 (2018).

30. Gu S, Fang L, Xu X. Using SOAPaligner for Short Reads Alignment. Curr Protoc Bioinformatics 44, 1111 11-17 (2013).

31. Wood DE, Lu J, Langmead B. Improved metagenomic analysis with Kraken 
454 32. Peng Y, Leung HC, Yiu SM, Chin FY. IDBA-UD: a de novo assembler for 455 single-cell and metagenomic sequencing data with highly uneven depth. 456 Bioinformatics 28, 1420-1428 (2012).

457 33. Uritskiy GV, DiRuggiero J, Taylor J. MetaWRAP-a flexible pipeline for 458 genome-resolved metagenomic data analysis. Microbiome 6, 158 (2018).

459 34. Olm MR, Brown CT, Brooks B, Banfield JF. dRep: a tool for fast and 460 accurate genomic comparisons that enables improved genome recovery from 461 metagenomes through de-replication. ISME J 11, 2864-2868 (2017).

462 35. Parks DH, Imelfort M, Skennerton CT, Hugenholtz P, Tyson GW. CheckM: 463 assessing the quality of microbial genomes recovered from isolates, single 464 cells, and metagenomes. Genome Res 25, 1043-1055 (2015).

465 36. Jain C, Rodriguez RL, Phillippy AM, Konstantinidis KT, Aluru S. High 466 throughput ANI analysis of $90 \mathrm{~K}$ prokaryotic genomes reveals clear species 467 boundaries. Nat Commun 9, 5114 (2018).

468 37. Chaumeil PA, Mussig AJ, Hugenholtz P, Parks DH. GTDB-Tk: a toolkit to 469 classify genomes with the Genome Taxonomy Database. Bioinformatics, 470 (2019).

471 38. Parks DH, Chuvochina M, Chaumeil PA, Rinke C, Mussig AJ, Hugenholtz P. 472 A complete domain-to-species taxonomy for Bacteria and Archaea. Nat 473 Biotechnol 38, 1079-1086 (2020).

474 39. He Z, Zhang H, Gao S, Lercher MJ, Chen WH, Hu S. Evolview v2: an online 475 visualization and management tool for customized and annotated 476 phylogenetic trees. Nucleic Acids Res 44, W236-241 (2016).

477 40. Besemer J, Lomsadze A, Borodovsky M. GeneMarkS: a self-training method 478 for prediction of gene starts in microbial genomes. Implications for finding 479 sequence motifs in regulatory regions. Nucleic Acids Res 29, 2607-2618 $480 \quad$ (2001).

481 41. Seemann T. Prokka: rapid prokaryotic genome annotation. Bioinformatics 30, 482 2068-2069 (2014).

483 42. Fu L, Niu B, Zhu Z, Wu S, Li W. CD-HIT: accelerated for clustering the 484 next-generation sequencing data. Bioinformatics 28, 3150-3152 (2012). 
485

486

487

488

489

490

491

492

493

494

495

496

497

498

499

500

501

502

503

504

505

506

507

508

509

510

\section{1}

512

513

514 201804010338),

515 Science and Technology Program of Guangdong Province (2014B020201002), the

43. Kanehisa M, Furumichi M, Tanabe M, Sato Y, Morishima K. KEGG: new perspectives on genomes, pathways, diseases and drugs. Nucleic Acids Res 45, D353-D361 (2017).

44. Lombard V, Golaconda Ramulu H, Drula E, Coutinho PM, Henrissat B. The carbohydrate-active enzymes database (CAZy) in 2013. Nucleic Acids Res 42, D490-495 (2014).

45. Chen L, Zheng D, Liu B, Yang J, Jin Q. VFDB 2016: hierarchical and refined dataset for big data analysis--10 years on. Nucleic Acids Res 44, D694-697 (2016).

46. Alcock BP, et al. CARD 2020: antibiotic resistome surveillance with the comprehensive antibiotic resistance database. Nucleic Acids Res 48, D517-D525 (2020).

47. Blin $\mathrm{K}$, et al. antiSMASH 4.0-improvements in chemistry prediction and gene cluster boundary identification. Nucleic Acids Res 45, W36-W41 (2017).

48. Crits-Christoph A, Diamond S, Butterfield CN, Thomas BC, Banfield JF. Novel soil bacteria possess diverse genes for secondary metabolite biosynthesis. Nature 558, 440-444 (2018).

49. Navarro-Munoz JC, et al. A computational framework to explore large-scale biosynthetic diversity. Nat Chem Biol 16, 60-68 (2020).

50. Shannon P, et al. Cytoscape: a software environment for integrated models of biomolecular interaction networks. Genome Res 13, 2498-2504 (2003).

51. Chaudhari NM, Gupta VK, Dutta C. BPGA- an ultra-fast pan-genome analysis pipeline. Sci Rep 6, 24373 (2016).

\section{ACKNOWLEDGEMENTS}

12 This work was supported by grants from National Natural Science Foundation of

13 China (No. 32002287), Guangzhou Municipal Science and Technology Bureau (No. 
516 Presidential Foundation of Guangdong Academy of Agricultural Sciences (grant

517 number 201918), Grant from Guangzhou Municipal Science and Technology Bureau

518 (No. 202102080151), and Guangdong Provincial Key Laboratory of Genome Read

519 and Write (No. 2017B030301011). We also thank the colleagues at BGI-Shenzhen for

520 sample collection, and discussions, and China National Genebank (CNGB) Shenzhen

521 for DNA extraction, library construction, and sequencing.

\section{AUTHOR CONTRIBUTIONS}

523 Conceived and designed the experiments: Y.Z. and W.W. Performed the experiments:

524 B.D, Y.Z., X.L, and X.J. Analyzed the data: Y.Z., X.L, T.H., J.Z., J.C, and G.L.

525 Contributed reagents/materials/analysis tools: B.D., Y.Z., X.J., Z.C., J.L., and L.X.

526 Supervised the work: L.X., B.W., Y.H., S.L., and J.L. Wrote the paper: B.D., Y.Z., X.L,

527 and T.H. Revised the paper: B.D., Y.Z., and W.W. All authors commented on the

528 manuscript.

COMPETING FINANCIAL INTERESTS

530 The authors declare no competing financial interests.

\section{DATA AVAILABILITY STATEMENT}

532 The data that support the findings of this study have been deposited into CNGB

533 Sequence Archive $(\mathrm{CNSA})^{21}$ of China National GeneBank DataBase $(\mathrm{CNGBdb})^{22}$

534 with accession number CNP0002075 and CNP0002072 for metagenomic and

535 bacterial genomic data, respectively. All the bacterial strains in our collection have

536 been deposited in China National GeneBank (CNGB), a non-profit,

537 public-service-oriented organization in China.

\section{ONLINE METHODS}

\section{Sample collection and bacteria culturing}

54142 healthy piglets (Duroc $\widehat{x} \times($ LandracexYorkshire) ㅇ) with good mental state were

542 selected for sampling, of which 12 piglets of lactation were sampled on 18 days for 
543 ileum and colon content, respectively, 21 weaned piglets were sampled on 35 days for

544 ileum and colon content, and 9 weaned piglets were sampled on 60 days for faeces.

545 During sampling, the intestinal contents were obtained by dissecting the colon and

546 ileum with sterile instruments. Every 3 samples from one line with same time point

547 and region were pooled together. All samples were stored at $-80 \square$ and prepared for

548 bacterial culturing and metagenome sequencing.

549 For bacteria culture, samples were processed according to our previous bacteria

550 culture-based study ${ }^{23}$. To acquire more different bacterial taxa, we applied a batch of

551 rich and selective culture medium which are provided in the Supplementary method.

552 The purified bacteria were maintained with $20 \%$ glycerine at $-80 \square$. Detailed

553 information about the origin of all strains, including the age of the donors and gut

554 region are presented in Supplementary Data 1.

555 The acquired bacteria were subject to $16 \mathrm{~S}$ rRNA gene sequencing. The amplification 556 of $16 \mathrm{~S}$ rRNA gene was using $27 \mathrm{f}$ and $1492 \mathrm{r}$ primers and the obtained sequences were 557 trimmed as previously described ${ }^{24}$. The taxonomy of bacteria was recognized by blast 558 the 16S rRNA gene sequences against reference sequences within the NCBI 16S 559 ribosomal RNA sequence database. We used the recommended threshold of $98.7 \%$ 560 and $94.5 \%$ as the species and genus boundaries ${ }^{25}$. 16S rRNA gene sequences were 561 aligned by MAFFT v7.310 ${ }^{26}$ and trimmed by trimAl v1.4. rev $22^{27}$ with auto option. 562 Phylogenetic tree based on 16S rRNA gene sequences was reconstructed by using the 563 maximum-likelihood method with FastTree Version 2.1.3 SSE3 ${ }^{28}$.

\section{Genome sequencing and assembly}

565 Genomic DNA of the 226 representative strains was extracted using the method as 566 previously described ${ }^{23}$. The draft genomes were sequenced for $100 \mathrm{bp}$ paired-end on 567 the DNBSEQ-T7 platform. The raw reads were filtered using SOAPnuke26 $6^{29}$ (v1.5.0; 568 -1 20 -q 0.4 -n 0.1 -d -M 3 --seqType 0 -Q 2 -c 2.66666666666667). After preliminary 569 assessment of the genome size based on kmer, SPAdes (v3.11.1; -T 4 -m 100) was 570 used to assembly. 


\section{Metagenome sequencing, assembly, and binning}

572 Metagenomic DNA from 14 samples was extracted and sequenced according to the

573 method described above. Raw reads of each sample were preprocessed by

574 SOAPnuke (v1.5.2) ${ }^{29}$ using the 'filter' module (option -1 20 -q 0.2 -n 0.05 -Q 2 -d -c

$575 \quad 0-50-7$ 1), and host reads were removed by SOAPaligner ${ }^{30}$ (v2.22, option -m 4 -s

57630 -r 1 -n 50 -x 1000 -v 4). Kraken2 $2^{31}$ was used to assign taxonomic labels to the

577 metagenome reads and calculate bacterial abundance and the profile was provided in

578 Supplementary Table 1. Shannon alpha diversity indexes ('diversity’ function) and

579 Bray-Curtis dissimilarity indexes ('vegdist' function) for the principal coordinate

580 analysis (PCoA) were identified in R software with species abundances.

581 Thereafter, IDBA-UD (v 1.1.3) ${ }^{32}$ was used to assemble and merge the optimal contig.

582 MAGs were generated using three different tools configured in the 'binning' module

583 of metaWRAP (v1.1.5) ${ }^{33}$ (integrated with MaxBin2, MetaBAT2, and CONCOCT)

584 and were finally consolidated and optimized using 'Bin_refinement'. dRep ${ }^{34}$ (v2.5.4,

585 option -p 8 -comp 70 -con 5) was used to de-redundancy and preliminary quality

586 control of the MAGs. The whole-genome assembly was as described by Zou et $a l^{23}$.

587 The quality of the MAGs and isolated genomes was estimated by CheckM

$588(\mathrm{v} 1.0 .12)^{35}$. The classification criteria for high-quality genomes are based on $>90 \%$

589 completeness and $<5 \%$ contamination, while genomes with $<70 \%$ completeness

590 or $>5 \%$ contamination were not included in the following analysis.

\section{Species-level clustering, phylogenetic and taxonomic analyses}

592 FastANI (v1.32) ${ }^{36}$ was used to calculate the pairwise ANI, and genomes were

593 clustered into species-level clusters with a 95\% ANI cut-off using the R package

594 hclust. dRep was used to select the best genome from each cluster as the

595 representative. All genomes were taxonomically annotated using GTDB-Tk ${ }^{37}$

596 (v1.3.0, database release $95^{38}$ ). Any lineage without an annotated species or invalid

597 names with reserved suffixes was considered to represent a potential new species.

598 The maximum-likelihood phylogenetic tree of the representative genomes was 
599 constructed with GTDB-Tk ('infer' followed by 'classify_wf'). The phylogenetic

600 tree was viewed using the online tool EVOLVIEW v2 ${ }^{39}$.

601 Alignment with other genome collections

602 We downloaded the genomes from two studies, which represent the reference 603 dataset for the MAGs and the isolated genome of the pig gut microbiome. Wang et $604 a{ }^{9}{ }^{9}$ generated a total of 360 substantially complete MAGs (>70\% completeness, <5\% 605 contamination) as the first metagenomic reference for swine intestinal microbiota. 606 PiBAC $^{12}$ comprised 117 high-quality isolated genomes representing 110 species. 607 Both datasets were downloaded from NCBI (BioProject: PRJNA494875 and 608 PRJNA561470, respectively). We performed a pairwise ANI calculation for all 609 genomes, and only those ANI $>95 \%$ were considered to be matched.

\section{Gene prediction and function annotation}

611 GeneMark.hmm PROKARYOTIC $(\mathrm{v} 3.38)^{40}$ is a gene prediction program for 612 metagenomes. Protein-coding sequences (CDS) for each genome were predicted and 613 annotated by Prokka v1.14.6 $6^{41}$. The metagenome non-redundancy gene catalog was 614 generated by CD-HIT v4.6.3 ${ }^{42}$ (cd-hit-est with option -c 0.95 defining protein 615 identity of $95 \%$ ). Based on $95 \%$ protein similarity, we clustered the gene catalog 616 with $\mathrm{Xiao}^{5}$ and calculated the gene overlap of the metagenome and genome.

617 Functional characterization of all CDS was performed by BLAST analysis with the 618 KEGG database (release 81) ${ }^{43}, \mathrm{CAZyDB}^{44}$ (downloaded from http://www.cazy.org/, 619 as of April 2016), and VFDB (setB, 2021-07) ${ }^{45}$. ARGs were identified with rgi 620 (5.2.0) using reference data from the Comprehensive Antibiotic Resistance Database 621 (CARD, v 3.1.2)

622 The SMBGs were identified by using anti-SMASH (v4.2.0 ${ }^{47}$ and were clustered by 623 using MIBiG (version 1.4) ${ }^{48}$ with reference BGCs by BiG-SCAPE ${ }^{49}$ with default 624 parameters. The similarity networks of the SMBGs were displayed by using 625 Cytoscape (v3.8.2) ${ }^{50}$. 


\section{Pan genome analysis of the representative species}

627 The Bacterial Pan Genome Analysis tool (BPGA) pipeline ${ }^{51}$ was used for protein

628 clustering (usearch, $80 \%$ identity value), identification of core, accessory, and

629 unique genes of each cluster, and generation of pan-genome accumulation curves for

630 the 171 genomes in eight clusters. Clusters of Orthologous Groups (COGs) of

631 proteins based on Prokka annotation described above were collected to identify the

632 core COGs specific to genome clusters with an in-house script. Total ARGs of these

633 clusters were summarized to determine the presence and absence of them in each

634 genome and to identify the core and dispensable genomes of antibiotic resistance

635 with an in-house script.

\section{Detecting the production of reuterin}

637 Reuterin production from $L$. reuteri cultures was determined according to the

638 previous described method ${ }^{18}$. In brief, cell pellets were harvested by centrifugation

639 from overnight grown cultures of L. reuteri in MRS broth. The pellets were washed

640 twice with $0.1 \mathrm{M}$ potassium phosphate buffer, resuspended in $70 \%$ glycerol, and

641 then incubated at $25^{\circ} \mathrm{C}$ for $2.5 \mathrm{~h}$ under anaerobic conditions. Quantification of

642 reuterin in the suspension was determined by the colorimetric method as described ${ }^{18}$. 
657 Fig. 1 | Gut microbiota composition in the ileum, colon, and faeces of weanling 658 piglets. a-b, Comparison of the phylum-level proportional abundance of microbiota. 659 c, Comparison of Shannon alpha diversity index of microbiota. d, Principal 660 coordinates analysis (PCoA) of the bacterial composition at the species level based 661 on Bray-Curtis dissimilarity.

662 Fig. 2 | Phylogeny of 266 genomes from isolated bacterial strains and 482 663 non-redundant metagenomic assembly genomes (MAGs). a, Phylogenetic tree 664 comprising the 428 species-level clusters. Novel clusters are highlighted by red clades, 665 and the phylum is displayed in the first outer layer. Blue and orange bars in the second 666 outer layer represent the number of isolated genomes and MAGs in each cluster. b-c, 667 The number of MAGs and isolated genomes clusters matching PiBAC ${ }^{12}$ and Wang et $668 a l^{9}$, respectively.

669 Fig. 3 | Expansion of the present pig gene catalog. a, Coverage of the gene catalog 670 from Xiao et al. ${ }^{5}$ by different samples from this study. The order is arranged from low 671 to high coverage, and samples from the ileum, colon, and faeces are colored as blue, 672 green, and orange, respectively. b, The overlap between genes in all samples (red) and 673 the Xiao et al. gene catalog (blue). c, Overlap of genes between three approaches 674 (metagenome, cultured-base, and MAGs).

675 Fig. 4 | Functional repertoires of 748 genomes. a, The distribution of CAZymes 676 across different phylum. b. The distribution of ARGs in each sample and genome. The 677 presence and absence of ARGs is marked in blue and white, respectively. c, The 678 distribution of 19 different types of SMBGs at phylum level. d, The sequence 
679 similarity network of SMBGs and the MiBiG references. Each node represents a

680 SMBG, the color of the node indicates the species from which the genome was

681 derived and the black circle indicates the reference BGC. Edges drawn between the

682 nodes correspond to pairwise distances. The figure shows only SMBGs for which

683 their distance from the reference is less than 0.3 .

684 Fig. 5 | The function distribution of genes in pangenome analysis of 8 species. a,

685 Accumulating fitting curves of pangenome gene family number in each cluster. $\mathbf{b}$, The

686 distribution of ARGs present in the core genomes (pink) and dispensable genomes

687 (blue). b, The distribution of genes involved in the biosynthesis of reuterin in the core

688 genomes (pink) and dispensable genomes (blue).

689

690 Supplementary Figure 1 | The proportion of reads that were and were not 691 classified at the species level.

692 Supplementary Figure 2 | a-b, The comparison of genus-level proportional 693 abundance in the ileum, colon, and faeces of weanling piglets.

694 Supplementary Figure 3 | The 16S rRNA gene sequence phylogenetic tree of 695 1,476 strains. Novel clusters are highlighted by red clades. Phylum, sample, and 696 region are display in the first, second, and third outer layer, respectively.

697 Supplementary Figure 4 | The number of cultivated bacterial strains at genus 698 level from 14 samples.

699 Supplementary Figure 5 | Quality assessment of the genomes. a-b, Completeness 700 and contamination of 266 isolated genomes (a) and 482 MAGs (b), respectively.

701 Supplementary Figure 6 | The taxonomy of the 743 bacterial genomes. a-b, The 702 distribution of isolated genomes and MAGs across different phylum. Statistics show 703 the number of genomes (a) and clusters (b), respectively.

704 Supplementary Figure 7 | Comparison of MAGs and isolated genomes in 705 different quality standards. a, MAGs with $>70 \%$ completeness and $<5 \%$ 
706 contamination, while isolated genomes with $>90 \%$ completeness and $<5 \%$

707 contamination. b, Both MAGs and isolated genomes are high quality (with $>90 \%$

708 completeness and $<5 \%$ contamination).

709 Supplementary Figure 8 | The distribution of KEGG pathways in each genome.

710 The presence and absence of each pathway is indicated by blue and white, 711 respectively.

712 Supplementary Figure 9 | The distribution of VFDB in each genome. The 713 presence and absence of VFDB is indicated by blue and white, respectively.

714 Supplementary Figure 10 The accumulation curves of the pan and core genome

715 of the 8 representative clusters. Box plots indicate 25th and 75th percentiles with

716 medians shown as horizontal lines and whiskers set at 10th and 90th percentiles.

717 Supplementary Figure 11 | The gene family numbers of pan genome, core 718 genome and average genome of the 8 representative clusters. 

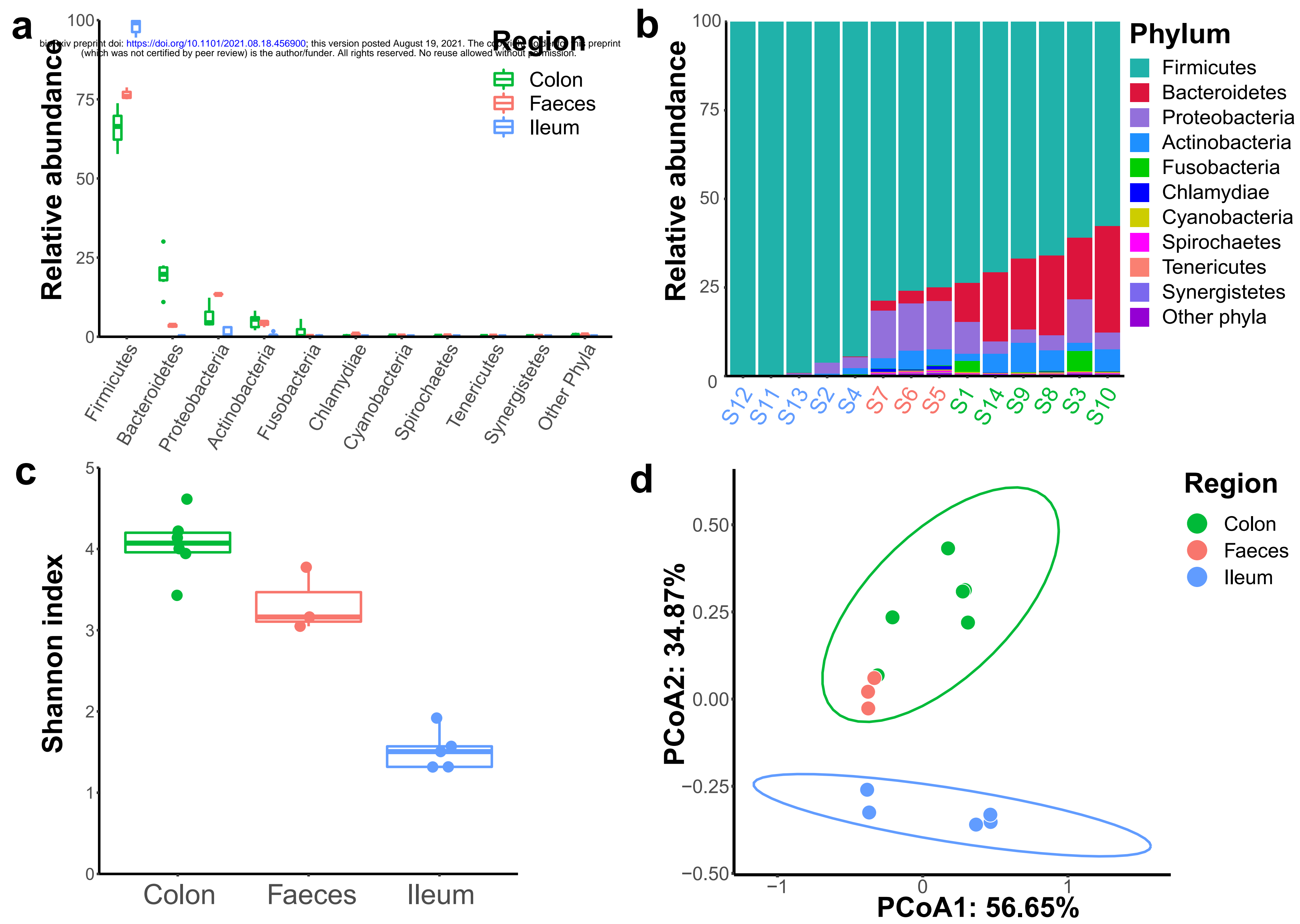

\section{Region}

Colon

Faeces

Ileum

Figure 1 
a

Phylum

Firmicutes

Bacteroidota

Proteobacteria

Spirochaetota

Actinobacteriota

Campylobacterota

Verrucomicrobiota

Desulfobacterota

Fusobacteriota

Myxococcota

\section{Number of genomes}

Isolated genomes

MAGs

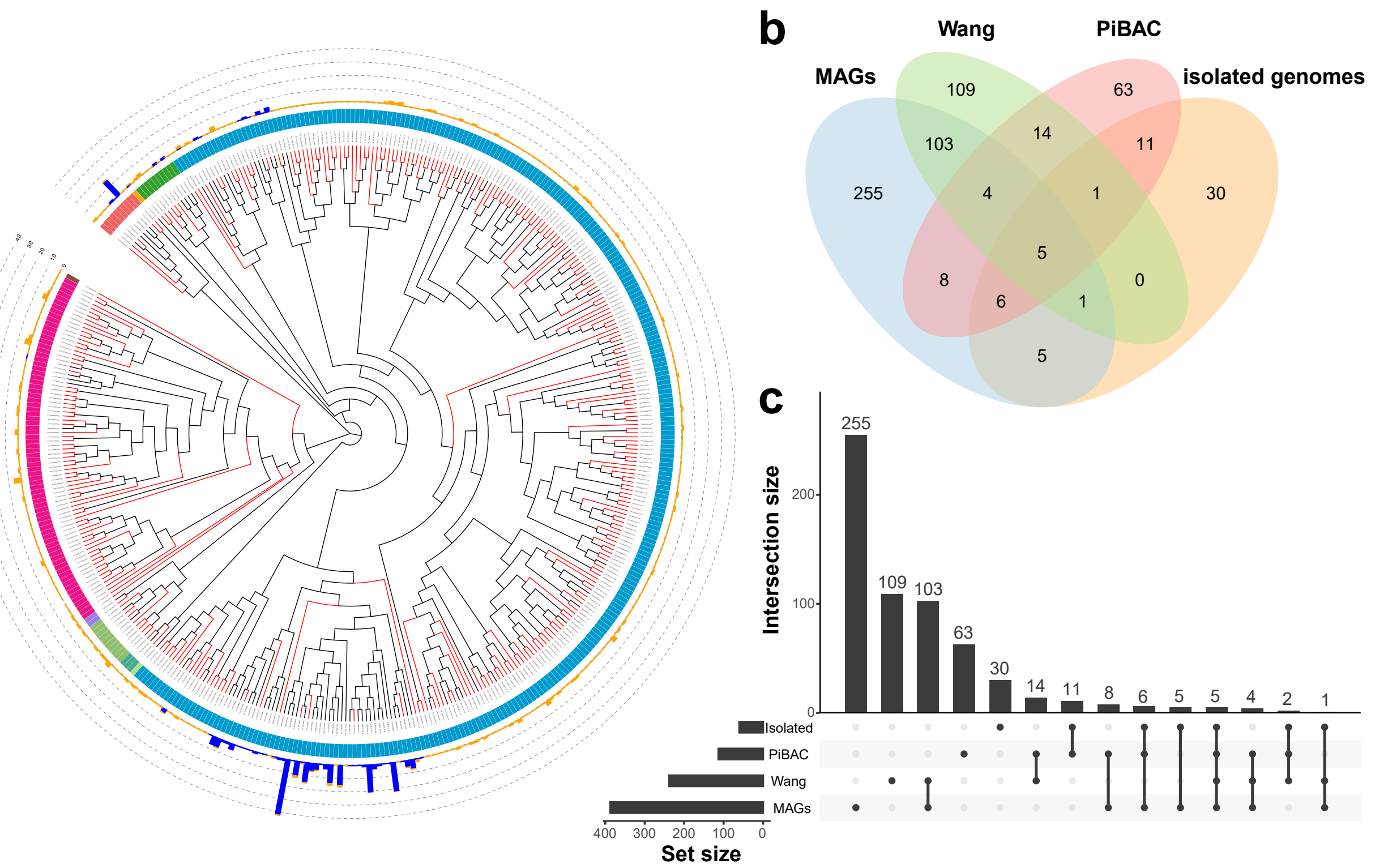

Figure 2 
a

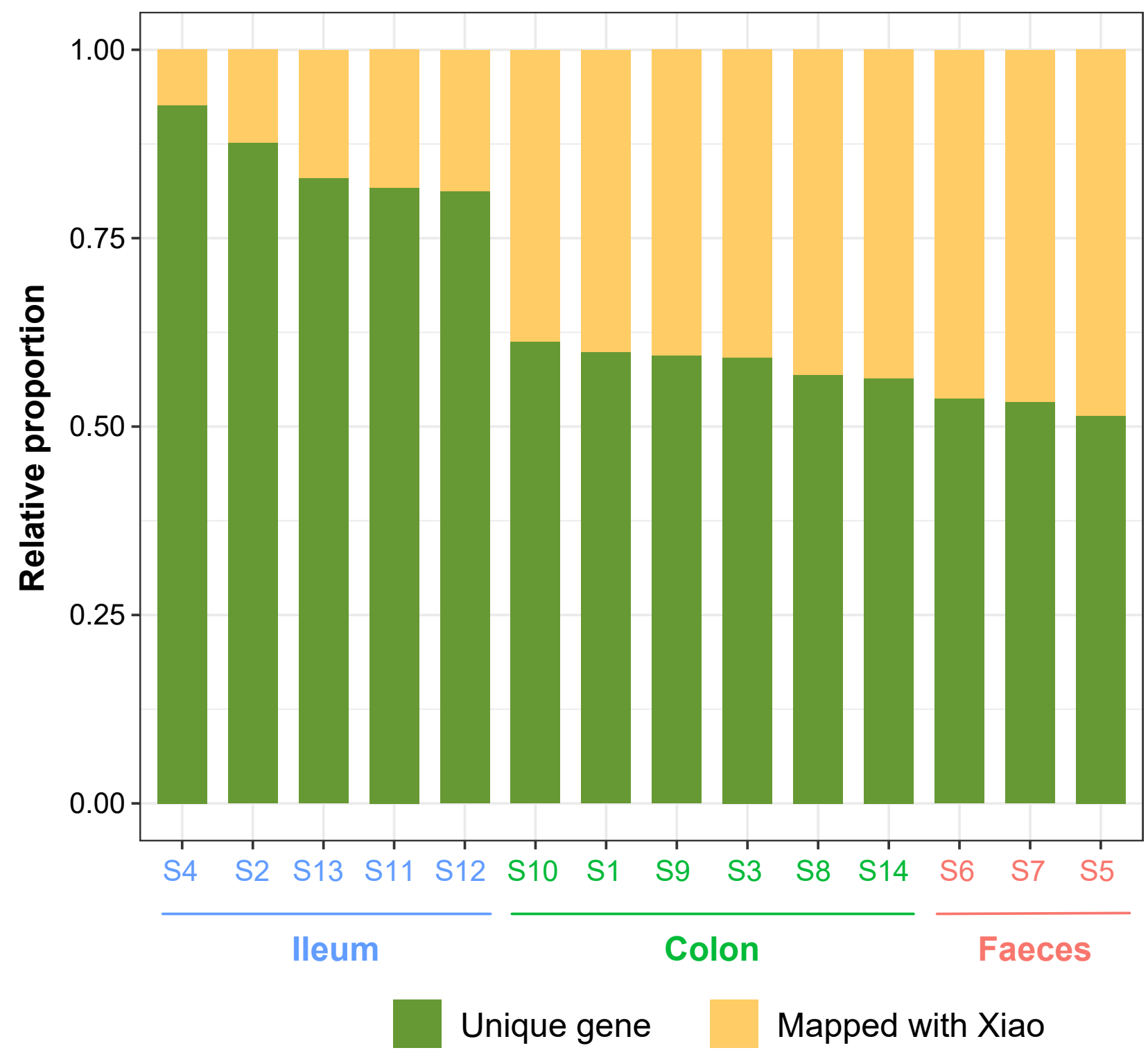

b

Xiao

Meta

C

Meta

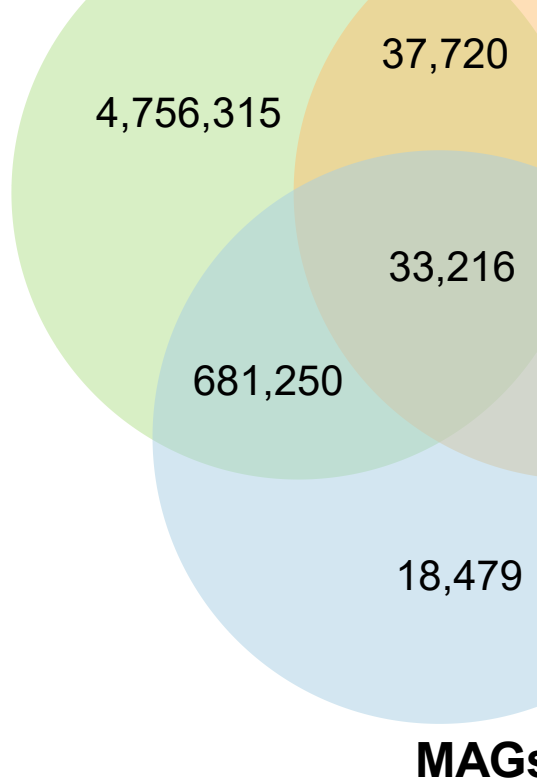

Isolated genomes
122,054

825

Figure 3 
a

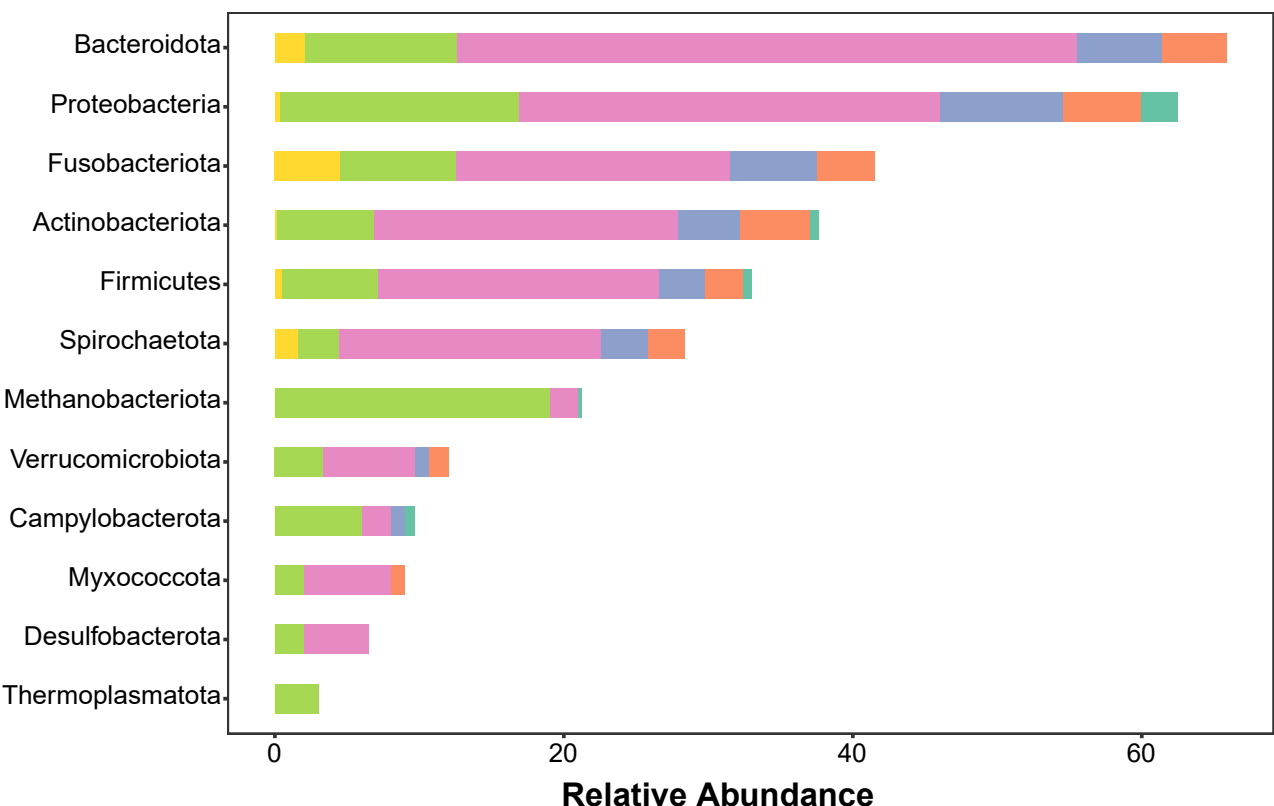

CAZYmes AAs CEs GTs CBMs GHs PLs

\begin{tabular}{|c|c|c|c|}
\hline Type & Meta & Isolated genomes & MAGs \\
\hline & Actinobacteriota & Bacteroidota & Campylobacterota \\
\hline & Desulfobacterota & Firmicutes & Fusobacteriota \\
\hline & Methanobacteri & Myxococcota & Proteobacteria \\
\hline & Spirochaetota & Verrucomicrobiota & Thermoplasmat \\
\hline
\end{tabular}

C
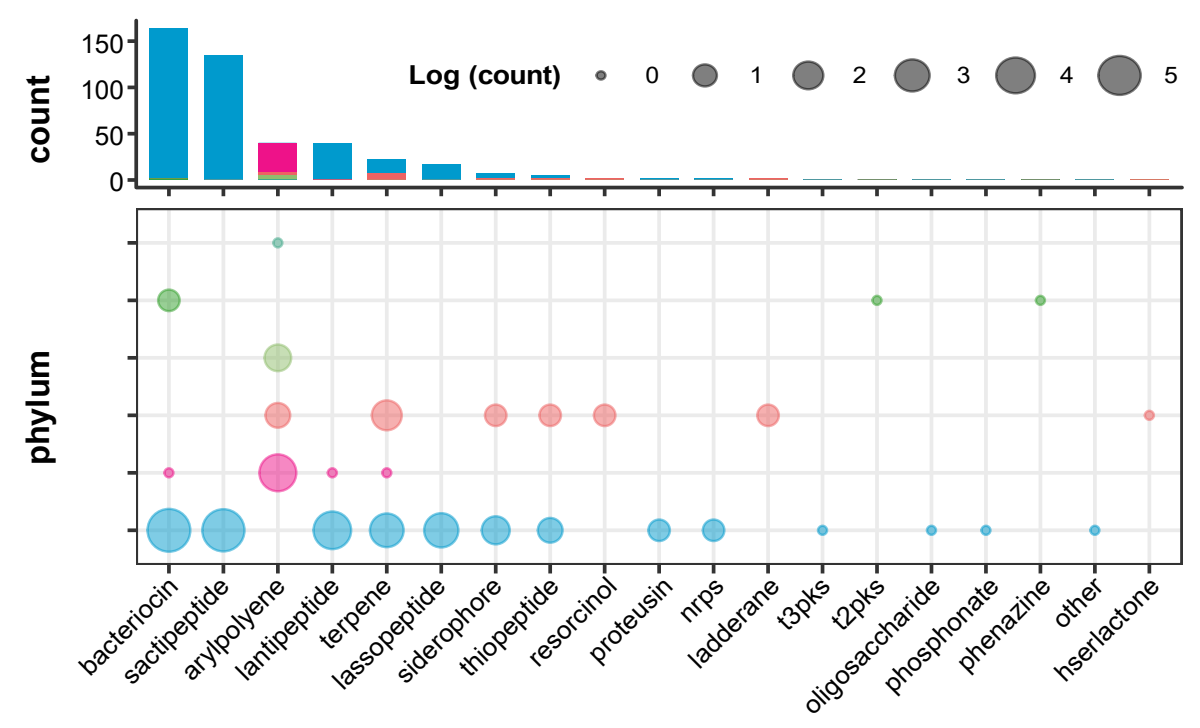

b

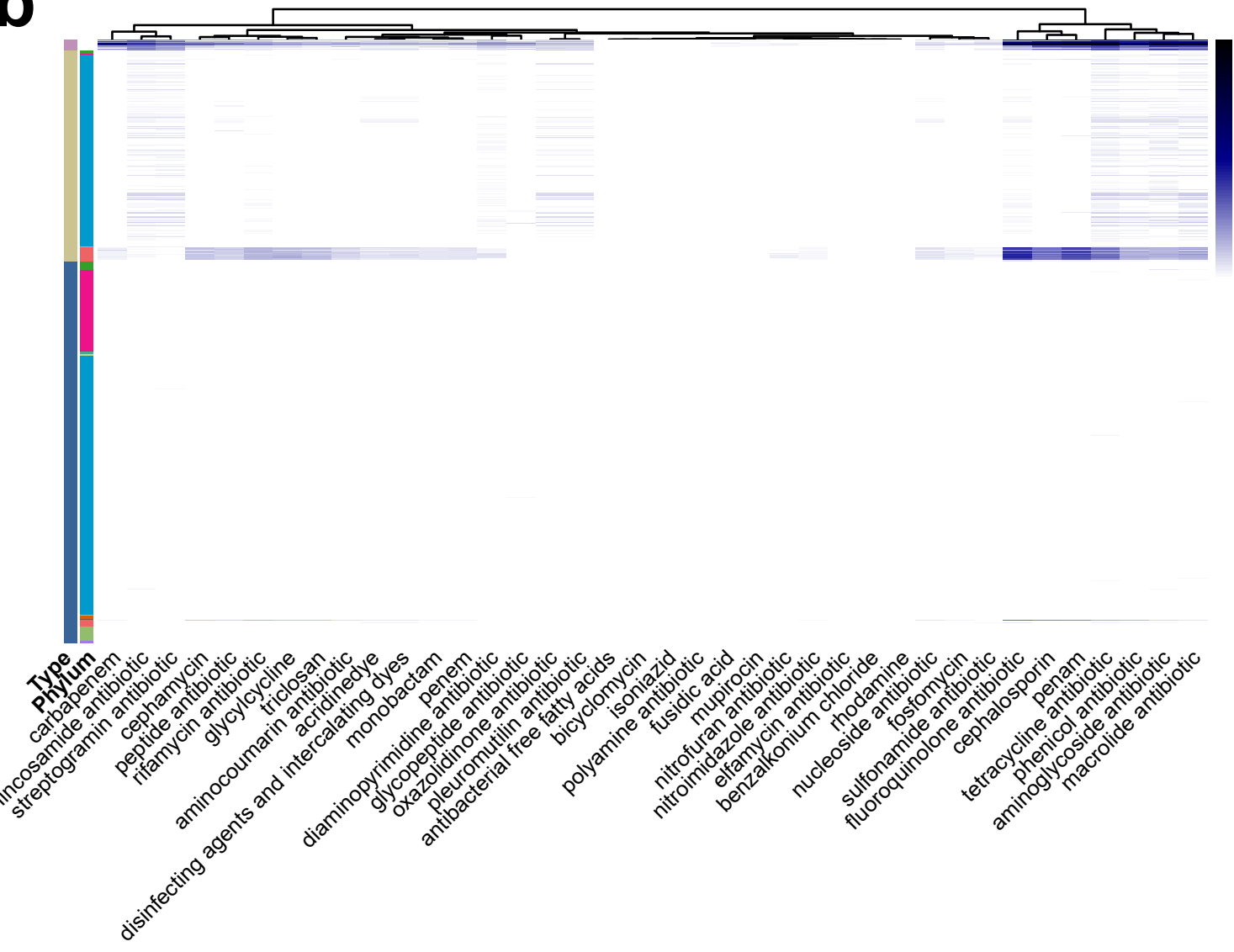

d

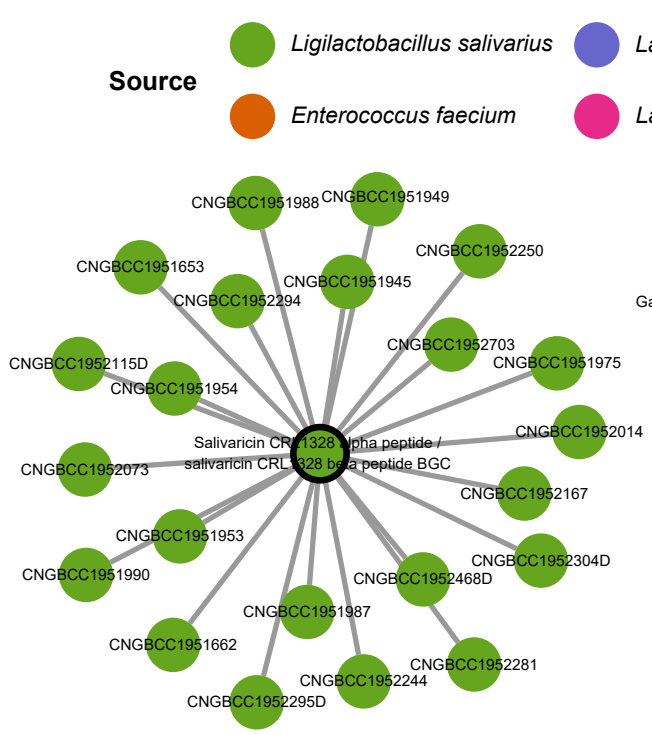

Lactobacillus gasseri

Enterococcus faecalis

Lactobacillus johnsonii

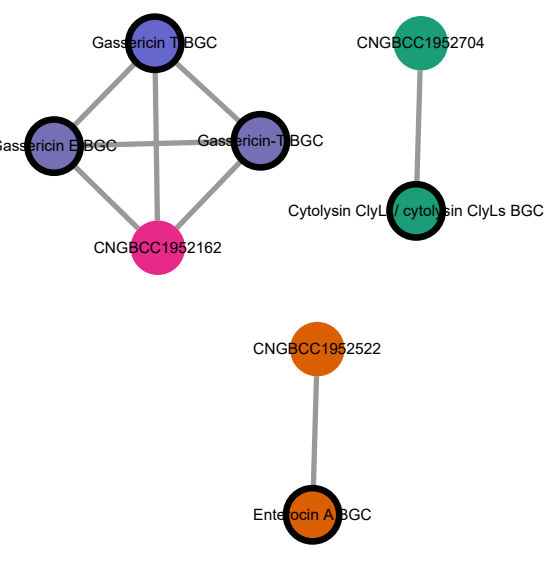

Figure 4 

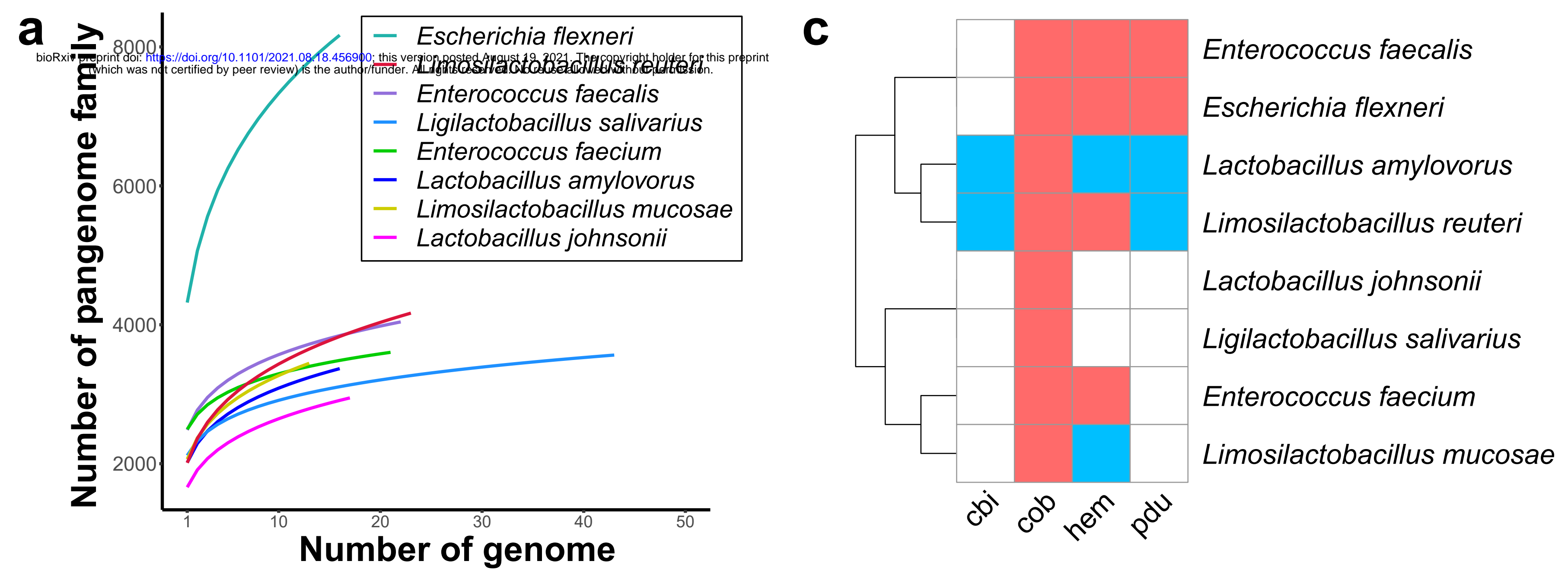

b



\section{Core genome}

Dispensable genome

\section{Escherichia flexneri}

Enterococcus faecium

Enterococcus faecalis

Lactobacillus amylovorus

Lactobacillus johnsonii

Limosilactobacillus reuteri

Ligilactobacillus salivarius

Limosilactobacillus mucosae 